\title{
Dengue-triggered Hemophagocytic lymphohistiocytosis in an adolescent with autoimmune thyroiditis
}

\author{
Muthukani Sankaranarayanan ${ }^{* 1}$, Suganya Velayutham ${ }^{2}$, Vani $^{3}$, Usharani $\mathrm{K}^{4}$ \\ 1 Consultant, Department of Neurosciences, Apollo Speciality Hospitals, Madurai, Tamil Nadu, India \\ 2 DNB Resident, Department of Medicine, Apollo Speciality Hospitals, Madurai, Tamil Nadu, India \\ 3 Senior Consultant Physician, Apollo Speciality Hospitals, Madurai, Tamil Nadu, India. \\ 4 Senior Consultant Pathologist, Apollo Speciality Hospitals, Madurai, Tamil Nadu, India.
}

*Corresponding Author: Dr. S. Muthukani MD, DM, Consultant, Department of Neurosciences, Apollo Speciality Hospitals, K.K. Nagar, Madurai-625020, Tamil Nadu, India

Email: mkani86@gmail.com; Tel: +91 - 9894293061

\begin{abstract}
Introduction: Hemophagocytic lymphohistiocytosis $(\mathrm{HLH})$ is a life threatening hyperinflammatory hematological syndrome characterized by cytokine storm and the diagnosis can be missed as it mimics sepsis or multi-organ dysfunction syndrome. Case Report: A 15-year old adolescent girl presented with six-day history of high grade intermittent fever with easy fatigability and dyspnea on exertion. She was found to have severe anemia elsewhere (Hemoglobin- $4 \mathrm{~g} / \mathrm{dl}$, normal 11-16.5 g/dl) and referred to our hospital due to persistent fever, tiredness and recurrent vomiting. On examination she was pale and febrile. Her blood investigations revealed moderate anemia, leukopenia and markedly elevated liver transaminases with coagulopathy. Dengue NS1 antigen and IgM antibodies were positive suggestive of acute dengue infection. Further workup revealed markedly elevated serum ferritin (37973 ng/ml, normal 10-120 ng/ml) and lactate dehydrogenase levels (5311 U/L, normal 180-360 U/L). Plasma fibrinogen level was low (164 mg/dl, normal 200-400 mg/dl). Bone marrow biopsy done on day 3 revealed hemophagocytosis. Serum TSH was mildly elevated $(6.5 \mathrm{mU} / \mathrm{L}$, normal $3.5-5 \mathrm{mU} / \mathrm{L})$ and anti-thyroid peroxidase (TPO) antibodies $(68 \mathrm{IU} / \mathrm{ml}$, normal <5.6 IU/ml) were also significantly elevated. Diagnosis of Hemophagocytic lymphohistiocytosis (HLH) secondary to dengue infection and autoimmune thyroiditis was made. She was treated with dexamethasone $10 \mathrm{mg} / \mathrm{m}^{2} /$ day from day 3 of admission along with supportive treatment. She rapidly improved and her laboratory parameters returned back to normal at 1 week of follow up. Conclusion: Dengue fever with unexplained severe anemia in the absence of bleeding should be evaluated for HLH as timely initiation of treatment will be life saving.
\end{abstract}

Keywords: Lifestyle Medicine, Five Star Doctor, Behavioral modification, Spirituality, Patient engagement, Physician burnout.

\section{INTRODUCTION}

Hemophagocytic lymphohistiocytosis (HLH) is an uncommon, yet life threatening hyperinflammatory hematological syndrome characterized by cytokine storm due to highly stimulated but ineffective immune process ${ }^{[1]}$. HLH can be primary or secondary. Primary HLH is familial and usually responds poorly to treatment including chemotherapy. Secondary HLH is triggered by various infections, autoimmune conditions, malignancy or immune deficiencies and responds well to treatment of underlying cause ${ }^{[2]}$. Epstein-Barr virus (EBV) and cytomegalovirus (CMV) infections are the common viral triggers of secondary $\mathrm{HLH}{ }^{[3]}$. Dengue infection may rarely trigger secondary HLH which, if goes unnoticed, and untreated may be fatal. We report an adolescent girl who presented with Dengue fever and autoimmune thyroiditis complicated by Hemophagocytic lymphohistiocytosis who made complete recovery with steroids.

\section{CASE REPORT}

A 15-year old adolescent girl presented with six-day history of high grade intermittent fever without chills or rigors but associated with body aches and arthralgias. She also had easy fatigability and dyspnea on exertion. She was initially evaluated elsewhere and found to have severe anemia (Hemoglobin- 4 $\mathrm{g} / \mathrm{dl}$, normal 11-16.5 g/dl). She was transfused with two units of packed cells and Vitamin B12 injection and referred to our hospital due to persistent fever, severe tiredness and recurrent 
vomiting. On examination at our hospital, she was febrile (Temperature- $101 \mathrm{~F}$ ), blood pressure-110/70 $\mathrm{mmHg}$, pulse rate-102/min and respiratory rate was $19 / \mathrm{min}$. She was pale. There was no rash, purpura or petechiae. Abdomen was soft with no palpable mass. Rest of the general physical examination was normal. Her initial blood counts revealed bicytopenia (Hemoglobin - $7.4 \mathrm{~g} / \mathrm{dl}$, normal 11-16.5 g/dl and leukocyte count $-2.7 \times 10^{9} / \mathrm{L}$, normal $4-11 \times 10^{9} / \mathrm{L}$ ) with low normal platelet count (Refer supplement Table-1 for serial laboratory parameters). Her liver function tests showed direct hyperbilirubinemia, transaminitis with coagulopathy (INR - 2, normal 0.9-1.1, APTT91 seconds, normal 23-33 seconds). She had marked elevation of transaminases to 100 times the normal values on day 3 . Her peripheral smear showed microcytic hypochromic RBCs with leukopenia and no evidence of malarial or filarial parasites. Ultrasonography of the abdomen showed mild hepatosplenomegaly, moderate ascites and bilateral pleural effusion. Dengue NS1 antigen and IgM antibodies were positive suggestive of acute Dengue infection. Further workup revealed markedly elevated serum ferritin (37973 ng/ml, normal 10-120 $\mathrm{ng} / \mathrm{ml}$ ) and lactate dehydrogenase levels (5311 U/L, normal 180 $360 \mathrm{U} / \mathrm{L})$. Workup for HLH was initiated. Plasma fibrinogen level was low (164 mg/dl, normal 200-400 mg/dl) and triglyceride level
(65 mg/dl, normal < $150 \mathrm{mg} / \mathrm{dl}$ ) was normal. Bone marrow biopsy done on day 3 of admission revealed hemophagocytosis (Figure-1). Serum TSH was mildly elevated $(6.5 \mathrm{mU} / \mathrm{L}$, normal 3.5-5 mU/L) and her T3 and T4 levels were normal. Serum antithyroid peroxidase (TPO) antibodies $(68 \mathrm{IU} / \mathrm{ml}$, normal $<5.6$ $\mathrm{IU} / \mathrm{ml}$ ) were also significantly elevated. Anti-nuclear antibody(ANA) by indirect immunofluorescence was negative. Serological tests for HIV, Hepatitis-B and Hepatitis-C were negative. Serum soluble CD-25 levels and NK cell function studies could not be tested. Diagnosis of Hemophagocytic lymphohistiocytosis (HLH) secondary to Dengue infection and autoimmune thyroiditis was made by serological evidence of acute Dengue infection, bicytopenia, very high ferritin, liver dysfunction, hypofibrinogenemia, splenomegaly, bone marrow evidence of hemophagocytosis and elevated antithyroid antibodies. She was treated with Dexamethasone injection 10 $\mathrm{mg} / \mathrm{m}^{2} /$ day from day 3 of admission along with fresh frozen plasma for coagulopathy. She rapidly improved, was afebrile on day 4 . Her blood counts and liver function parameters also returned back to normal after 1 week of follow up. She was switched over to oral prednisolone $(1 \mathrm{mg} / \mathrm{kg} / \mathrm{day})$ after one week and it was tapered over next 2 weeks.

Table 1: Serial hematological and biochemical parameters in the patient who presented with dengue-triggered HLH.

\begin{tabular}{|l|l|l|l|l|l|l|l|l|}
\hline Lab parameters & Normal values & Day 1 & Day 2 & Day 3 & Day 4 & Day 5 & Day 7 & Day 15 \\
\hline Hemoglobin (g/dl) & $11.5-16.5$ & 7.4 & 7.9 & 7.9 & 6.7 & 6.3 & 7.2 & 10.9 \\
\hline PCV (\%) & $37-47$ & 25 & 27 & 26 & 24 & 23 & 27 & 36 \\
\hline TLC (x 109/L) & $4-11$ & 2.7 & 8.8 & 14.9 & 4.6 & 5.1 & 6.7 & 7.2 \\
\hline Platelets (x 109/L) & $150-400$ & 170 & 150 & 220 & 340 & 410 & 420 & 480 \\
\hline T.Bil (mg/dl) & $0.2-1.1$ & 1.8 & 2.2 & 2.0 & 1.9 & 1.4 & 0.9 & 0.8 \\
\hline D. Bil (mg/dl) & $0-0.2$ & 1.6 & 1.8 & 1.6 & 1.4 & 0.9 & 0.6 & 0.5 \\
\hline AST (IU/L) & $5-45$ & 799 & 1340 & 4586 & 1831 & 662 & 102 & 42 \\
\hline ALT (IU/L) & $5-45$ & 308 & 471 & 1447 & 747 & 511 & 266 & 177 \\
\hline ALP (IU/L) & $<187$ & 106 & 115 & 105 & 111 & 100 & 85 & 81 \\
\hline Albumin (g/dl) & $3.5-5$ & 3.3 & 3.0 & 2.9 & 3.1 & 3.3 & 3.9 & 3.9 \\
\hline APTT (seconds) & $23-33$ & 90.5 & 71.7 & 59.2 & 42.8 & - & 27.2 & - \\
\hline INR & $0.9-1.1$ & 1.99 & - & 1.81 & - & - & - & - \\
\hline $\begin{array}{l}\text { Serum } \\
\text { (ng/ml) }\end{array}$ & $10-120$ & 37973 & - & - & - & - & - & 328 \\
\hline LDH (U/L) & $180-360$ & 5311 & - & 6532 & - & - & - & - \\
\hline
\end{tabular}

Legend: PCV- Packed cell volume; TLC- Total leukocyte count; T Bil- Total bilirubin; D Bil- Direct bilirubin; AST- Aspartate amino transferase; ALT- Alanine amino transferase; ALP- Alkaline phosphatase; APTT- Activated partial thromboplastin time; INR- International normalised ratio; $\mathrm{LDH}$ - Lactate dehydrogenase

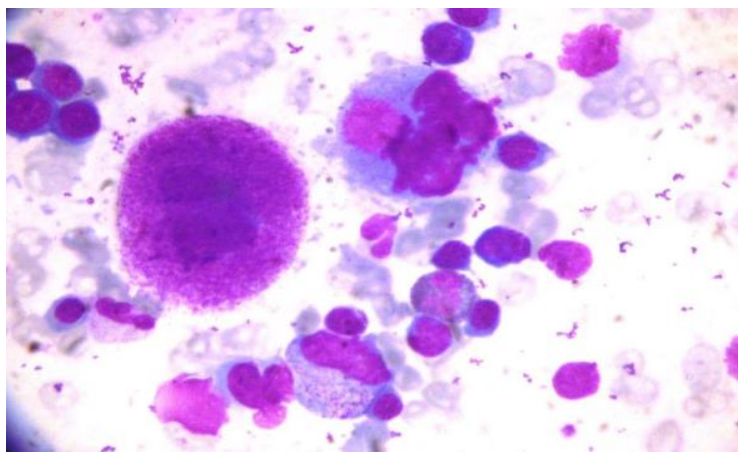

Legend for Figure 1: Arrow showing engulfment of hematopoietic cell (hemophagocytosis) by a macrophage on bone marrow aspiration smear (10x 100x magnification)

\section{DISCUSSION}

Hemophagocytic lymphohistiocytosis (HLH) is an uncommon hematological syndrome with uncontrolled immune activation that can be lethal due to multi-organ system failure, especially if untreated. HLH can be genetic or sporadic. Several infections, autoimmune disorders and/or malignancy can trigger secondary $\mathrm{HLH}$. This syndrome is clinically characterized by fever, organomegaly, effusions and its laboratory findings include cytopenia, high serum ferritin, lactate dehydrogenase and triglycerides, and deranged liver function including coagulopathy [4]. The revised guidelines for diagnosis of $\mathrm{HLH}$ by the histioytic society requires either molecular diagnosis consistent with $\mathrm{HLH}$ or the presence of five out of eight criteria, namely, fever, splenomegaly, cytopenias, hyperferritinemia, hypertriglyceridemia or hypofibrinogenemia, hemophagocytosis in bone marrow or other tissue, increase in sCD25 or 
reduced/absent NK cell function ${ }^{[5]}$.

The pathophysiology of HLH is poorly understood. There is unchecked inflammation leading onto activation of $T$ cells and macrophages with failure of natural killer cell function causing defective clearance of antigen presenting cells. Subsequently there will be release of high levels of cytokines and chemokines such as interleukin-1 (IL-1), IL-6, tumor necrosis factor and interferon- $\gamma$ into the circulation causing tissue damage in multiple organs with phagocytosis of hematopoietic elements ${ }^{[6]}$. Before the advent of treatment HLH was invariably fatal. HLH 2004 protocol recommends etoposide with dexamethasone as standard of care in both primary and acquired forms of HLH ${ }^{[5]}$. Intravenous immunoglobulin, anti-thymocyte globulin with subsequent hematopoietic stem cell transplantation (HSCT), monoclonal antibodies against IL-2R or IFN and alemtuzumab are tried in refractory cases ${ }^{[7]}$. In patients with defined infection triggered $\mathrm{HLH}$, appropriate therapy against inciting infection is recommended.

Dengue is caused by mosquito-borne flavivirus, commonly occurring in tropical and subtropical countries ${ }^{[8]}$. It can present with various clinical manifestations ranging from subclinical infection to life threatening Dengue hemorrhagic fever (DHF) or Dengue shock syndrome (DSS). Those who become infected with the virus for second time (Secondary dengue infection) are at greater risk of developing severe disease ${ }^{[9]}$. Dengue triggered $\mathrm{HLH}$, commonly described in children with complicated dengue infection, and usually presents in the second week of illness [1011]. Fever during dengue infection usually lasts for 3-7 days and persistent fever beyond 8 days with cytopenia should alert the clinician about the possibility of HLH. Leuopenia and thrombocytopenia are common in acute dengue infection. They usually develop hemoconcentration with elevation in hemoglobin and hematocrit from day 3 to day 8 unless complicated by bleeding [12]. Our patient did not have any evidence of DHF or DSS, yet had drop in hemoglobin. Moderate to severe anemia in the absence of internal or external bleeding with very high ferritin made us to suspect $\mathrm{HLH}$ from the first day of her presentation here. She also had another predisposing condition for $\mathrm{HLH}$, autoimmune thyroiditis which was also recognized during the same admission. How the dengue infection triggers HLH is not very clear. The possibility is high viral load activates large number of non- reactive $T$ cells and releases enormous amount of cytokines into the blood stream which, in the presence of another predisposing condition, results in a vicious cycle [13]. Review of the existing literature about treatment of Dengue-triggered HLH showed that some cases recovered with only supportive treatment [14-15]. We started her on steroids as she had progressive worsening of liver function tests, but she had very good response to the given treatment and made an uneventful recovery.

\section{CONCLUSION}

Our case report highlights that HLH may be an atypical presentation of an acute Dengue infection, especially with an underlying autoimmune condition such as autoimmune thyroiditis. The diagnosis of HLH can be missed as its clinical manifestations might be explainable by severe Dengue infection or sepsis with multi-organ dysfunction. We conclude that Dengue fever with unexplained severe anemia in the absence of bleeding should be evaluated for $\mathrm{HLH}$ as timely initiation of treatment will be lifesaving.

Conflict of interest: None to declare.

\section{REFERENCES}

1. Janka G, Zur Stadt U. Familial and acquired hemophagocytic lymphohistiocytosis. Hematol Am Soc Hematol Educ Program. 2005; 82-8.

2. Lakhotia M, Pahadiya HR, Gandhi R, Prajapati GR, Choudhary A. Stuck with pancytopenia in dengue fever: Evoke for hemophagocytic syndrome. Indian J Crit Care Med Peer-Rev Off Publ Indian Soc Crit Care Med. 2016; 20:55-6.

3. Schram AM, Comstock P, Campo M, Gorovets D, Mullally A, Bodio K, et al. Haemophagocytic lymphohistiocytosis in adults: a multicentre case series over 7 years. $\mathrm{Br} J$ Haematol. 2016; 172:412-9.

4. Aricò M, Janka G, Fischer A, Henter JI, Blanche S, Elinder G, et al. Hemophagocytic lymphohistiocytosis. Report of 122 children from the International Registry. FHL Study Group of the Histiocyte Society. Leukemia. 1996; 10:197-203.

5. Henter J-I, Horne A, Aricó M, Egeler RM, Filipovich AH, Imashuku $\mathrm{S}$, et al. HLH-2004: Diagnostic and therapeutic guidelines for hemophagocytic lymphohistiocytosis. Pediatr Blood Cancer. 2007; 48:124-31.

6. Usmani GN, Woda BA, Newburger PE. Advances in understanding the pathogenesis of $\mathrm{HLH}$. Br J Haematol. 2013; 161:609-22.

7. Janka GE, Lehmberg K. Hemophagocytic syndromes--an update. Blood Rev. 2014; 28:135-42.

8. Guzman MG, Harris E. Dengue. Lancet Lond Engl. 2015; 385:453-65.

9. Martina BEE, Koraka $P$, Osterhaus ADME. Dengue virus pathogenesis: an integrated view. Clin Microbiol Rev. 2009; 22:564-81.

10. Ray S, Kundu S, Saha M, Chakrabarti P. Hemophagocytic Syndrome in Classic Dengue Fever. J Glob Infect Dis. 2011; 3:399-401.

11. Khurram M, Faheem M, Umar M, Yasin A, Qayyum W, Ashraf A, et al. Hemophagocytic Lymphohistiocytosis Complicating Dengue and Plasmodium vivax Coinfection. Case Rep Med. 2015; 696842.

12. Chaloemwong J, Tantiworawit A, Rattanathammethee T, Hantrakool S, Chai-Adisaksopha C, Rattarittamrong E, et al. Useful clinical features and hematological parameters for the diagnosis of dengue infection in patients with acute febrile illness: a retrospective study. BMC Hematol. 2018; 18:20.

13. Halstead SB. Pathogenesis of Dengue: Dawn of a New Era. F1000Research. 2015; 4.

14. Lu P-L, Hsiao H-H, Tsai J-J, Chen T-C, Feng M-C, Chen T-P, et al. Dengue virus-associated hemophagocytic syndrome and dyserythropoiesis: a case report. Kaohsiung J Med Sci. 2005; 21:34-9.

15. Wan Jamaludin WF, Periyasamy P, Wan Mat WR, Abdul Wahid SF. Dengue infection associated hemophagocytic syndrome: Therapeutic interventions and outcome. J Clin Virol Off Publ Pan Am Soc Clin Virol. 2015; 69:91-5. 